\title{
Extension of WAF type methods to non-homogeneous Shallow Water Equations with pollutant
}

\author{
E. D. Fernández-Nieto, G. Narbona-Reina* \\ July 27, 2015
}

\begin{abstract}
This paper deals with the extension of the WAF method to discretize Shallow Water Equations with pollutants. We consider two different versions of the WAF method, by approximating the intermediate waves using the flux of HLL or the direct approach of HLLC solver. It is seen that both versions can be written under the same form with different definitions for the approximation of the velocity waves. We also propose an extension of the method to non-homogeneous systems. In the case of homogeneous systems it is seen that we can rewrite the third component of the numerical flux in terms of an intermediate wave speed approximation. We conclude that - in order to have the same relation for non-homogeneous systems - the approximation of the intermediate wave speed must be modified. The proposed extension of the WAF method preserves all stationary solutions, up to second order accuracy, and water at rest in an exact way, even with arbitrary pollutant concentration. Finally, we perform several numerical tests, by comparing it with HLLC solver, reference solutions and analytical solutions.
\end{abstract}

Short title : the WAF method for non-homogeneous SWE with pollutant.

Keywords : Finite Volume Method, well-balanced, upwinding, shallow water, source terms, WAF, HLLC, pollutant.

Subject Classifications : AMS (MOS) : 65N06, 76B15, 76M20, 76N99.

\section{Introduction}

In this paper we study the extension of the WAF (Weighted Average Flux) method to Shallow Water Equations (SWE in what follows) with topography and transport of pollutants.

*Departamento de Matemática Aplicada I, E.T.S. Arquitectura. Universidad de Sevilla. Avda. Reina Mercedes 2. 41012 Sevilla, Spain (edofer@us.es, gnarbona@us.es). 
This method was introduced by Toro in [29]. WAF is a one-step method of Godunovtype to solve hyperbolic conservation laws that achieves second order accuracy by averaging the solution of the conventional Riemann problem with piece-wise constant initial data.

There are many works related to the applications of the WAF method, for example to the Shallow Water and Euler equation (see [30, 31]). The extension to the multidimensional case was performed by Billet and Toro in [3]. In [25] the WAF method is combined with WENO reconstructions.

As it is well known, due to Godunov's theorem, linear schemes with high order accuracy generate spurious oscillations near large gradients of the solution. To avoid this problem, the WAF method is used with a flux limiter function, getting a non-linear TVD (Total Variation Diminishing) scheme of second order accuracy. This property, and the fact that it does not need characteristic decomposition, make the WAF method a quick solver with a good order of accuracy for solving Shallow Water equations with pollutant.

With respect to high order numerical schemes, we can find the ADER (AdvectionDiffusion-Reaction) approach, developed by Toro et al. (See for example [33]). The ADER is a finite-volume Godunov-type approach that allows to obtain schemes with arbitrary order of accuracy, involving the use of data reconstruction and a first order flux solver. We can also find the extension of ADER to the multidimensional case $[24,37,27,36]$ and to the non-homogeneous case [36, 39, 34].

Regarding the WAF method, we can find in [35] the introduction of the ADER-TVD approach. In this version of ADER methods a TVD flux is used to construct the ADER scheme instead of the conventional use of a first-order monotone flux. Specifically, the WAF method is taken as the only direct method of second order accuracy. In the case of no reconstruction, the ADER-WAF scheme provides a second order TVD scheme, obtaining a superior scheme to the conventional ADER.

There are different ways of extending a numerical scheme to non-homogeneous hyperbolic systems. The pioneering work of Roe [23] relates the choice of a quadrature formula for the source term with the property of preserving stationary solutions. Different extensions have been done: see for instance Bermudez-Vázquez [2], Greenberg-Leroux [16], LeVeque [19], Zhou et al. [41], Chacón et al. [8], Perthame-Simeoni [22]. These techniques focus on the problem of a hyperbolic system with source term (or balance law).

Another different form to tackle the problem is to rewrite the balance law as a hyperbolic system in non-conservative form by adding a new equation. For example, for SWE with topography, if $z_{b}$ denotes the function corresponding to the bed of the channel, the equation $\partial_{t} z_{b}=0$ is added. Then a numerical scheme for the non-conservative system is applied and finally the scheme is rewritten in function of the original unknowns. See for instance LeFloch [18], Parés-Castro [21], Gosse [15].

In this paper we adopt the strategy of studying the problem in form of a balance law. We follow the technique introduced in [12] where the concept of 'asymptotically wellbalanced method' is introduced. The purpose is for the method to preserve all stationary solutions up to second order over a set whose measure of the complementary tends to zero, when the space step also tends to zero (see Definition 3.1). To perform the extension of the method we must localize the corresponding numerical diffusion that must be neglected 
for stationary solutions.

The extension of the WAF method put forward in this paper is actually asymptotically well-balanced. Moreover it exactly preserves water at rest. The case of water at rest is the only stationary solution that allows to have an arbitrary pollutant concentration, it also introduces an additional difficulty in order for this solution to be preserved.

In [17] Kurganov and Petrova present a central-upwing scheme using a second order state reconstruction verifying that the gradient of the height of the water column at intercells coincides with the gradient of the free water surface. Using this reconstruction it is proved that the proposed method is positivity preserving. Other positivity preserving techniques applied to VFROE with hydrostatic reconstruction [1] are introduced in [20] and $[5]$.

We cannot prove the method positivity preserving for general situations. We have not found negative values in the numerical tests. Specifically in Tests 4, 5 and 6 dry/wet fronts and vacuum generation appear. Using a dry/wet technique it is possible to prove positivity of the height in this front, in some cases by using a reduced CFL condition. For the tests to be developed in Section 4 we use the dry/wet treatment proposed in [6]. It consists of a local redefinition of the topography in the intercells corresponding to dry/wet transition. Another dry/wet technique is presented in [7] considering the Godunov flux only in the intercell corresponding to the transition. The nonlinear Rieman problem whose solution depends on the tail of the jump in the bottom is solved. In [13] this technique is generalized to be used with high-order finite volume methods through state reconstructions.

Another important issue is that although the scheme is well-balanced, a bad approximation of the pollutant concentration may be obtained, as it is shown in Test 1. We observe that the problem arises from the definition of the approximation of the intermediate wave speed. In fact in this work we show that the third component of the numerical flux for the WAF method can be rewritten in terms of an approximation of the intermediate wave speed. In order to have the same property for the extended version of the WAF method for non-homogeneous systems we conclude that the approximation of the intermediate wave speed must be modified. In Test 1 we can actually see that we obtain a correct profile for pollutant concentration with this modified approximation of the intermediate wave speed.

After this introduction, the paper continues with Section 2, where we summarize how the WAF method is obtained for the homogeneous case. We consider two different versions corresponding to two different approaches for the intermediate flux and we rewrite them under an unique formulation. Later, in Section 3 we perform the extension of the method for SW equations with pollutant and topography. In Subsection 3.1 we summarize the technique introduced in [10] which is based in the study of the numerical diffusion introduced by the numerical scheme. In 3.2 we propose the extension of the WAF method for balance laws, in 3.3 the well-balanced properties of the proposed scheme are studied. Later, in 3.4 we present the intermediate wave speed depending on the definition of the source term. Finally in Section 4 several numerical tests are presented: a numerical study of the well-balanced properties of the method, three tests that involve the treatment of dry/wet fronts, another one to study the order of accuracy and a last one where we make a comparison with a reference solution when a periodic time-varying pollutant concentration 
is imposed as a boundary condition.

\section{The WAF method}

In this section we summarize the WAF method for the homogeneous SWE with pollutant. We begin by considering the homogeneous SWE given by the system

$$
\begin{cases}\frac{\partial W}{\partial t}+\frac{\partial F(W)}{\partial x}=0, & x \in[0, L], t \in[0, T], \\ W(x, 0)=W_{0} & x \in[0, L] ;\end{cases}
$$

where

$$
W=\left(\begin{array}{c}
h \\
q \\
r
\end{array}\right), \quad F(W)=\left(\begin{array}{c}
q \\
\frac{q^{2}}{h}+\frac{1}{2} g h^{2} \\
\frac{q r}{h}
\end{array}\right),
$$

the unknowns are $h$, the height of the water column, $q$, the discharge; if we denote by $\psi$ the pollutant concentration, or another passive scalar, then $r=h \psi . W_{0}$ is the initial data, $L$ is the length of the domain, $T$ the final time and $g$ is the constant gravity.

We consider a partition of the domain $\left\{x_{i}\right\}_{i}=\{i \Delta x\}_{i}$ where, by simplicity, we take $\Delta x$ a constant space step, and we denote by $t^{n}=t^{n-1}+\Delta t$ the time values, with $\Delta t$ the time step. If we use a finite volume method in conservative form to approximate the solution of this problem, we have

$$
W_{i}^{n+1}=W_{i}^{n}-\frac{\Delta t}{\Delta x}\left(\phi_{i+1 / 2}^{n}-\phi_{i-1 / 2}^{n}\right)
$$

where we denote by $W_{i}^{n}$ an approximation of the mean value of the solution on the control volume $\left(x_{i-1 / 2}, x_{i+1 / 2}\right)$ at time $t=t^{n}$ :

$$
W_{i}^{n} \approx \frac{1}{\Delta x} \int_{x_{i-1 / 2}}^{x_{i+1 / 2}} W\left(x, t^{n}\right) d x
$$

and by $\phi_{i+1 / 2}^{n}=\phi\left(W_{i}^{n}, W_{i+1}^{n}\right)$ the numerical flux function that characterize each method.

As we have mentioned before, WAF type methods have second order accuracy. This is obtained by averaging the solution of the associated Riemann problem in time and space. We summarize the form in which it is obtained.

We consider the Riemann problem associated to (1) with initial data $W_{i}^{n}$ and $W_{i+1}^{n}$ :

$$
\left\{\begin{array}{l}
\frac{\partial W}{\partial t}+\frac{\partial F(W)}{\partial x}=0 \\
W(x, 0)= \begin{cases}W_{i} & x<0 \\
W_{i+1} & x>0\end{cases}
\end{array}\right.
$$




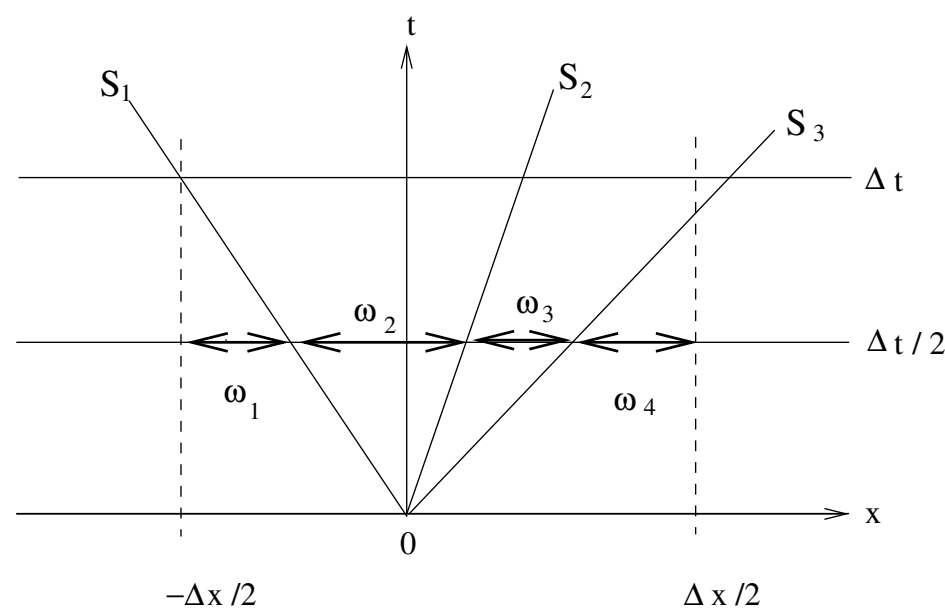

Figure 1: Computational grid and intermediate waves to compute the WAF method.

We remove $n$ superindex for simplycity of notation.

We denote by $S_{i}$ for $1 \leq i \leq 3$ the approximation of the characteristic velocities. We consider a computational grid $\left[x_{1}, x_{2}\right] \times\left[t_{1}, t_{2}\right]$ in the $x-t$ plane, and take $t_{1}=0, t_{2}=\Delta t$, $x_{1}=-\Delta x / 2$ and $x_{2}=\Delta x / 2$ (see Figure 1). Then, the numerical flux of the WAF method is obtained by integrating in this volume. By using the midpoint rule for the time integral, that is, by evaluating the flux at $t=\Delta t / 2$, we get:

$$
\phi_{i+1 / 2}^{W A F}=\frac{1}{\Delta x} \int_{-\Delta x / 2}^{\Delta x / 2} F\left(\tilde{W}\left(x, \frac{\Delta t}{2}\right)\right) d x .
$$

Being $\tilde{W}$ the solution of the Riemann problem.

We denote by $\omega_{k}$ the measure of the interval $k$ between the waves $S_{k-1}$ and $S_{k}$ divided by $\Delta x$ (see Figure 1), given by:

$$
\omega_{k}=\frac{1}{2}\left(c_{k}-c_{k-1}\right), c_{0}=-1, c_{N+1}=1, \text { with } c_{k}=\frac{\Delta t}{\Delta x} S_{k},
$$

where $N$ is the number of waves. Concretely, $N=3$ for SWE with pollutant. So, we can write the numerical flux as follows:

$$
\phi_{i+1 / 2}^{W A F}=\sum_{k=1}^{N+1} w_{k} F_{i+1 / 2}^{(k)},
$$

with $F_{i+1 / 2}^{(k)}$ the value of the flux function in the interval $k$.

If we expand the expression (4), having into account the values of $\omega_{k}$, we obtain the next expression for the WAF flux:

$$
\phi_{i+1 / 2}^{W A F}=\frac{1}{2}\left(F_{i}+F_{i+1}\right)-\frac{1}{2} \sum_{k=1}^{N} c_{k} \Delta F_{i+1 / 2}^{(k)},
$$


where we have denoted by $\Delta F_{i+1 / 2}^{(k)}=F_{i+1 / 2}^{(k+1)}-F_{i+1 / 2}^{(k)}$, and $F_{i}$ is the flux function at point $x_{i}$.

The WAF scheme is a second order accuracy method in time and space, so according to Godunov's theorem, it produces spurious oscillations near great gradients of the solution. To overcome this fact, a TVD stabilization is taken. If we denote by $B(v)$ a flux limiter function, then the limiter function is defined by

$$
A(v, c)=1-(1-|c|) B(v) .
$$

So the TVD-WAF flux function becomes as follows:

$$
\phi_{i+1 / 2}^{W A F}=\frac{1}{2}\left(F_{i}+F_{i+1}\right)-\frac{1}{2} \sum_{k=1}^{N} M_{k} \Delta F_{i+1 / 2}^{(k)},
$$

where

$$
M_{k}=\operatorname{sign}\left(S_{k}\right) A_{k} .
$$

Some suitable choices for $A_{k}$ can be found in [32]. We have used in particular the Van Albada's limiter:

$$
A_{k}=A\left(v^{(k)}, c_{k}\right)=\left\{\begin{array}{ll}
1 & \text { if } v^{(k)} \leq 0 \\
1-\frac{\left(1-\left|c_{k}\right|\right) v^{(k)}\left(1+v^{(k)}\right)}{1+v^{(k)^{2}}} & \text { if } v^{(k)} \geq 0
\end{array},\right.
$$

where

$$
v^{(k)}=\left\{\begin{array}{l}
\frac{p_{i}^{(k)}-p_{i-1}^{(k)}}{p_{i+1}^{(k)}-p_{i}^{(k)}} \quad \text { if } c_{k}>0 \\
\frac{p_{i+2}^{(k)}-p_{i+1}^{(k)}}{p_{i+1}^{(k)}-p_{i}^{(k)}} \quad \text { if } c_{k}<0
\end{array},\right.
$$

being $p$ a scalar value. In our case we set $p=h+z_{b}$ for $k=1,3$ (waves $S_{1}$ and $S_{3}$ ) and $p=\psi$ for $k=2\left(\right.$ wave $\left.S_{2}\right)$.

the WAF method depends on the choice of an approximation for the intermediate waves. The usual choice is the HLLC flux (see [32]). This method is constructed from the HLL method. The improvement of HLLC vs. HLL is that in flux approximation it has into account the intermediate eigenvalue (corresponding to the wave $S_{2}$ ) which is associated to the third unknown, so it gives a better approximation for this variable.

The numerical flux function for HLLC scheme can be written as follows:

$$
\begin{aligned}
& {\left[\phi_{i+1 / 2}^{H L L C}\right]_{(j)}=\left[\phi_{i+1 / 2}^{H L L}\right]_{(j)} \quad j=1,2 ;} \\
& {\left[\phi_{i+1 / 2}^{H L L C}\right]_{(3)}=\left[\phi_{i+1 / 2}^{H L L}\right]_{(1)} \psi_{*} \quad \text { where } \psi_{*}= \begin{cases}\psi_{i} & \text { if } S_{2} \geq 0 \\
\psi_{i+1} & \text { if } S_{2}<0 .\end{cases} }
\end{aligned}
$$

Where the HLL flux is defined as:

$$
\phi_{i+1 / 2}^{H L L}= \begin{cases}F_{i} & \text { if } S_{1} \geq 0 ; \\ F^{H L L}=\frac{S_{3} F_{i}-S_{1} F_{i+1}+S_{3} S_{1}\left(W_{i+1}-W_{i}\right)}{S_{3}-S_{1}} & \text { if } S_{1} \leq 0 \leq S_{3} \\ F_{i+1} & \text { if } 0 \geq S_{3} .\end{cases}
$$


We can find different possibilities for $S_{1}$ and $S_{3}$ (see [32]), for instance we can set:

$$
S_{1}=u_{i}-\sqrt{g h_{i}} \quad S_{3}=u_{i+1}+\sqrt{g h_{i+1}} .
$$

There are also several choices for $S_{2}$, we take the following value:

$$
S_{2}=\frac{S_{1} q_{i+1}-S_{3} q_{i}-S_{1} S_{3}\left(h_{i+1}-h_{i}\right)}{q_{i+1}-q_{i}-h_{i+1} S_{3}+h_{i} S_{1}} .
$$

This approximation of the intermediate speed $S_{2}$ is proposed by Toro in [32] for HLLC solver.

Remark 2.1 There are several possibilities to estimate $S_{2}$. The definition of $S_{2}$ must be an approximation of the velocity in the usually called 'star region', that is, the region defined between $S_{1}$ and $S_{3}$ waves in Figure 1.

The approximation of the velocity at the star region can be done using a Riemann solver. We can find different possiblilities based on several properties. For example, the exact depth positivity preservation or the presence of two-shock or two-rarefaction waves, see [32] for details.

The choice of $S_{2}$ in equation (10) is presented in [32] for the homogeneous HLLC solver. This one is obtained by imposing that $h^{(2)}=h^{(3)}$, being $h^{(i)}, i=2,3$ the approximations of the height in regions $w_{2}$ and $w_{3}$ respectively (see Figure 1). Following [32] we can see that this value of $S_{2}$ has the property of being exact when one of the states is a dry bed state, because in this case $S_{2}$ is the speed of the front.

To compute the flux, since $F^{(2)}=F^{(3)}$ for the first and second components, we can use $F^{H L L}$, defined by equation (9). For the third component of the flux, following the HLLC approach, we can use $F^{H L L} \psi$ with $\psi=\psi_{i}$ at the left of $S_{2}$ and $\psi=\psi_{i+1}$ at the right of $S_{2}$. Then, we can write the WAF flux as follows:

$$
\begin{gathered}
{\left[\phi^{W A F}\right]_{(j)}=\left[\frac{F_{i}+F_{i+1}}{2}-\frac{1}{2}\left(M_{1}\left(F^{H L L}-F_{i}\right)+M_{3}\left(F_{i+1}-F^{H L L}\right)\right)\right]_{(j)} \text { for } j=1,2 .} \\
{\left[\phi^{W A F}\right]_{(3)}=\left[\frac{F_{i}+F_{i+1}}{2}\right]_{(3)}-\frac{1}{2}\left(M_{1}\left(\left[F^{H L L}\right]_{(1)} \psi_{i}-\left[F_{i}\right]_{(3)}\right)+M_{2}\left[F^{H L L}\right]_{(1)}\left(\psi_{i+1}-\psi_{i}\right)+\right.} \\
\left.+M_{3}\left(\left[F_{i+1}\right]_{(3)}-\left[F^{H L L}\right]_{(1)} \psi_{i+1}\right)\right) .
\end{gathered}
$$

Another variant that we consider in this paper is to set $F^{(2)}$ and $F^{(3)}$ equal to the definition of $\phi^{H L L}$ instead of $F^{H L L}$, obtaining a method with smaller dependence on the choice of the flux limiters. Although it is not possible to prove second order accuracy for this version 
of the WAF method, numerically we have obtained similar results for both versions. We obtain the following numerical flux in this case

$$
\left[\phi^{W A F-2}\right]_{(j)}= \begin{cases}{\left[\frac{F_{i}+F_{i+1}}{2}-\frac{1}{2} \frac{\Delta t}{\Delta x} M_{3}\left(F_{i+1}-F_{i}\right)\right]_{(j)}} & 0 \leq S_{1} \\ {\left[\phi^{W A F}\right]_{(j)}} & S_{1} \leq 0 \leq S_{3} ; \text { for } j=1,2 . \\ {\left[\frac{F_{i}+F_{i+1}}{2}-\frac{1}{2} \frac{\Delta t}{\Delta x} M_{1}\left(F_{i+1}-F_{i}\right)\right]_{(j)}} & 0 \geq S_{3} .\end{cases}
$$

and for the third component:

$$
\left[\phi^{W A F-2}\right]_{(3)}= \begin{cases}{\left[\frac{F_{i}+F_{i+1}}{2}\right]_{(3)}-\frac{1}{2} \frac{\Delta t}{\Delta x}\left[M_{3} \psi_{i+1}\left(F_{i+1}-F_{i}\right)+M_{1} F_{i}\left(\psi_{i+1}-\psi_{i}\right)\right]_{(1)} \quad 0 \leq S_{1} ;} \\ {\left[\phi^{W A F}\right]_{(3)} \quad S_{1} \leq 0 \leq S_{3} ;} \\ {\left[\frac{F_{i}+F_{i+1}}{2}\right]_{(3)}-\frac{1}{2} \frac{\Delta t}{\Delta x}\left[M_{1} \psi_{i}\left(F_{i+1}-F_{i}\right)+M_{3} F_{i+1}\left(\psi_{i+1}-\psi_{i}\right)\right]_{(1)} \quad 0 \geq S_{3} .}\end{cases}
$$

\subsection{Rewriting the WAF method}

We have presented two different versions of the numerical flux for the WAF method, corresponding to definitions (11)-(12) and (13)-(14). In this section we rewrite both versions of the WAF method under a compact formulation by introducing a generalization of the definitions of $S_{i}, i=1,2,3$.

From now on, with the purpose of clarity, we denote

$$
S_{L}=S_{1}, \quad S_{*}=S_{2}, \quad S_{R}=S_{3}, \quad \text { and analogously } \quad M_{L}=M_{1}, \quad M_{*}=M_{2} \quad M_{R}=M_{3} .
$$

We introduce the values $\bar{S}_{L}, \bar{S}_{*}, \bar{S}_{R}$. For the first version (11)-(12) these values coincide with their original values

$$
\bar{S}_{L}=S_{L}, \quad \bar{S}_{*}=S_{*}, \quad \bar{S}_{R}=S_{R}
$$

We can also see that the flux defined by (13)-(14) only varies in this definition. In this case we define the extended version of these values as follows:

$$
\bar{S}_{L}=\min \left\{S_{L}, 0\right\} ; \quad \bar{S}_{*}= \begin{cases}S_{L} & 0 \leq S_{L} \\ S_{*} & S_{L} \leq 0 \leq S_{R} ; \quad \bar{S}_{R}=\max \left\{S_{R}, 0\right\} . \\ S_{R} & S_{R} \leq 0 .\end{cases}
$$

These definitions allow us to write both versions of the WAF methods under the same compact expression

$$
\begin{aligned}
\phi_{(j)}^{W A F}=\left[\frac{F_{i}+F_{i+1}}{2}\right. & -\frac{1}{2} \frac{1}{\bar{S}_{R}-\bar{S}_{L}}\left(\left(M_{R} S_{R}-M_{L} S_{L}\right)\left(F_{i+1}-F_{i}\right)-\right. \\
& \left.\left.-\bar{S}_{R} \bar{S}_{L}\left(M_{R}-M_{L}\right)\left(W_{i+1}-W_{i}\right)\right)\right]_{(j)} ; \text { for } j=1,2 .
\end{aligned}
$$




$$
\begin{aligned}
\phi_{(3)}^{W A F}=\left[\frac{F_{i}+F_{i+1}}{2}\right]_{(3)} & -\frac{1}{2} \frac{1}{\bar{S}_{R}-\bar{S}_{L}}\left[\left(\bar{S}_{R} M_{R} \psi_{i+1}-\bar{S}_{L} M_{L} \psi_{i}\right)\left(F_{i+1}-F_{i}\right)+\right. \\
& +\bar{S}_{L} \bar{S}_{R}\left(M_{L} \psi_{i}-M_{R} \psi_{i+1}\right)\left(W_{i+1}-W_{i}\right)+ \\
& \left.+M_{*}\left(\psi_{i+1}-\psi_{i}\right)\left(\bar{S}_{R} F_{i}-\bar{S}_{L} F_{i+1}+\bar{S}_{L} \bar{S}_{R}\left(W_{i+1}-W_{i}\right)\right)\right]_{(1)} .
\end{aligned}
$$

In the following section we shall use this formulation to perform the extension to nonhomogeneous case.

\section{Extension to non-homogeneous system}

We consider now the non-homogeneous shallow-water equations with pollutant:

$$
\begin{cases}\frac{\partial W}{\partial t}+\frac{\partial F(W)}{\partial x}=G(x, W), & x \in[0, L], t \in[0, T], \\ W(x, 0)=W_{0} & x \in[0, L] .\end{cases}
$$

If we take the topography source term and we denote by $z_{b}(x)$ the height of the topography at point $x$, we have:

$$
G(x, W)=\left(\begin{array}{c}
0 \\
-g h z_{b}^{\prime}(x) \\
0
\end{array}\right)
$$

For the treatment of the non-homogeneous system, the usual technique is to approximate the numerical flux function by a numerical flux for the homogeneous system and to consider an upwind discretization of the source term (see [2]). Nevertheless, in order to obtain the structure of the numerical scheme in conservative form, we must integrate equation (19) on the control volume $\left(x_{i-1 / 2}, x_{i+1 / 2}\right)$. Thus, we obtain

$$
\frac{W_{i+1}^{n}-W_{i}^{n}}{\Delta t}+\frac{F_{i+1 / 2}-F_{i-1 / 2}}{\Delta x}=\frac{1}{\Delta x} \int_{x_{i-1 / 2}}^{x_{i+1 / 2}} G(x, W(x)) d x .
$$

Where, if we consider a Godunov solver, $F_{i+1 / 2}$ denotes an approximation of $F\left(\tilde{W}\left(x_{i+1 / 2}\right)\right)$ with $\tilde{W}$ the solution of the Riemann problem associated to the non-homogeneous system. The key point is that the Riemann problem depends on the original system, so it depends on the source term. Then, $F_{i+1 / 2}$ depends on the definition of the source term. So we use the following structure for the scheme to approximate (19):

$$
\frac{W_{i+1}^{n}-W_{i}^{n}}{\Delta t}+\frac{\phi_{G, i+1 / 2}^{n}-\phi_{G, i-1 / 2}^{n}}{\Delta x}=G_{C i}^{n}
$$


We have denoted by $\phi_{G}$ the numerical flux depending on the source term $G$ and by $G_{C i}$ a centered approximation of $G$ at point $x_{i}$ given by:

$$
G_{C i}=\frac{1}{2}\left(G_{i+1 / 2}-G_{i-1 / 2}\right)
$$

where

$$
G_{i+1 / 2}=\left(\begin{array}{c}
0 \\
-g h_{i+1 / 2} \frac{z_{b}\left(x_{i+1}\right)-z_{b}\left(x_{i}\right)}{\Delta x} \\
0
\end{array}\right) \text { with } h_{i+1 / 2}=\frac{h_{i+1}+h_{i}}{2} .
$$

So, the objective of this section is to define $\phi_{G}$, as a generalization of the WAF method depending on $G$. To do it, we focus on the well-balanced properties.

Following [10], firstly we must identify the numerical diffusion associated to the WAF method, and secondly we shall study how to define $\phi_{G}$ with the objective that the numerical diffusion terms were zero for all stationary solutions.

We summarize the idea of this technique in Subsection 3.1. Next, in 3.2 we apply it to extend WAF methods presented in Section 2. Finally in 3.3 we study their well-balanced properties. In Subsection 3.4 we present a generalization of the intermediate wave speed.

\subsection{Studying the numerical viscosity of the method}

The technique introduced in [10] is to study the numerical viscosity of the method to extend it to non-homogeneous system. The objective is that the numerical viscosity term must vanish for all stationary solutions.

We begin by considering the discretization of the homogeneous system

$$
\frac{\partial W}{\partial t}+\frac{\partial F(W)}{\partial x}=0
$$

We shall see in Subsection 3.2 that we can rewrite almost all components of the WAF method in the following form

$$
\phi_{i+1 / 2}=\frac{F_{i}+F_{i+1}}{2}+\nu_{1}(W)\left(W_{i+1}-W_{i}\right)+\nu_{2}(W)\left(F_{i+1}-F_{i}\right)
$$

for specific values of $\nu_{1}$ and $\nu_{2}$.

The numerical scheme defined by (2)-(23) can be viewed as a centered discretization of the equivalent system:

$$
\frac{\partial W}{\partial t}+\frac{\partial F(W)}{\partial x}+\Delta x\left[\frac{\partial}{\partial x}\left(\nu_{1}(W) \frac{\partial W}{\partial x}\right)+\frac{\partial}{\partial x}\left(\nu_{2}(W) \frac{\partial F(W)}{\partial x}\right)\right]=0 .
$$

Note that, if the Jacobian matrix of $F, A$, is not singular we can characterize the regular stationary solutions of the homogeneous system for being $W$ constant, so they are also solutions of the equivalent system (24). For non regular solutions, observe that by the 
Rankine-Hugoniot condition $\left(F\left(W_{R}\right)-F\left(W_{L}\right)=\xi\left(W_{R}-W_{L}\right)\right)$, for stationary shocks $(\xi=0)$ it is possible that $F(W)$ is constant, while $W$ may jump.

For the non-homogeneous case (19), if we consider a centered approximation for the source term, then the equivalent system is written as follows

$$
\frac{\partial W}{\partial t}+\frac{\partial F(W)}{\partial x}+\Delta x\left[\frac{\partial}{\partial x}\left(\nu_{1} \frac{\partial W}{\partial x}\right)+\frac{\partial}{\partial x}\left(\nu_{2} \frac{\partial F(W)}{\partial x}\right)\right]=G(x, W)
$$

but if we have a stationary solution $W$ of (19), it satisfies $\frac{\partial F(W)}{\partial x}=G(x, W)$, thus we can see that $W$ is not a stationary solution of (25).

If $W$ is a stationary solution of (19), and if we assume that the jacobian matrix of $F$, $A$, is not singular, then it satisfies:

$$
\frac{\partial F(W)}{\partial x}=G(x, W) \quad \text { and } \quad \frac{\partial W}{\partial x}=A^{-1}(W) G(x, W) .
$$

So, $W$ is a stationary solution of the equivalent system

$$
\begin{aligned}
\frac{\partial W}{\partial t}+ & \frac{\partial F(W)}{\partial x}+\Delta x\left[\frac{\partial}{\partial x}\left(\nu_{1}\left(\frac{\partial W}{\partial x}-A^{-1}(W) G(x, W)\right)\right)+\right. \\
+ & \left.\frac{\partial}{\partial x}\left(\nu_{2}\left(\frac{\partial F(W)}{\partial x}-G(x, W)\right)\right)\right]=G(x, W) .
\end{aligned}
$$

Finally, we consider a second approximation of (26), defined by (20) with the following definition of $\phi_{G}$,

$$
\begin{aligned}
\phi_{G, i+1 / 2} & =\frac{F_{i}+F_{i+1}}{2}+\Delta x\left[\nu_{1}\left(W_{i}, W_{i+1}\right)\left(\frac{W_{i+1}-W_{i}}{\Delta x}-\tilde{A}_{i+1 / 2}^{-1} G_{i+1 / 2}\right)+\right. \\
& \left.+\nu_{2}\left(W_{i}, W_{i+1}\right)\left(\frac{F_{i+1}-F_{i}}{\Delta x}-G_{i+1 / 2}\right)\right]
\end{aligned}
$$

where $G_{i+1 / 2}$ is defined in $(22)$ and $\tilde{A}_{i+1 / 2}^{-1}$ is an approximation of $A^{-1}\left(W_{i+1 / 2}\right),\left(W_{i+1 / 2}\right.$ is an intermediate state for $W_{i}$ and $\left.W_{i+1}\right)$ given by:

$$
\begin{aligned}
& \tilde{A}_{i+1 / 2}^{-1}=P \tilde{D}_{i+1 / 2}^{-1} P^{-1}, \text { where } P \text { is the eigenvectors matrix and } \\
& \tilde{D}_{i+1 / 2}^{-1}=\operatorname{diag}\left(l_{i}, i=1,2,3\right) \text { with } l_{i}= \begin{cases}\frac{1}{\lambda_{i}} & \text { if } \lambda_{i} \neq 0 \\
0 & \text { otherwise }\end{cases}
\end{aligned}
$$

being

$$
\lambda_{1}=\frac{q_{i+1 / 2}}{h_{i+1 / 2}}-\sqrt{g h_{i+1 / 2}}, \quad \lambda_{2}=\frac{q_{i+1 / 2}}{h_{i+1 / 2}}, \quad \lambda_{3}=\frac{q_{i+1 / 2}}{h_{i+1 / 2}}+\sqrt{g h_{i+1 / 2}} .
$$




\subsection{Extension to non-homogeneous system}

In Section 2.1 we have written the WAF method under a unique formulation given by equations (17) and (18). In this section we shall see that we can rewrite it under the structure (23) for first and second components. The third component includes another term that can be written in terms of $S_{*}$. Finally, following Subsection 3.1 we propose the extension of the WAF method to non-homogeneous systems.

Firstly we focus on the first two components, given by equation (17). Setting the following values for the viscosity coefficients, it can be written under form (23):

$$
\begin{aligned}
& \nu_{1}(W)=-\frac{1}{2} \frac{\bar{S}_{R} \bar{S}_{L}}{\bar{S}_{R}-\bar{S}_{L}}\left(M_{L}-M_{R}\right) \\
& \nu_{2}(W)=-\frac{1}{2} \frac{M_{R} \bar{S}_{R}-M_{L} \bar{S}_{L}}{\bar{S}_{R}-\bar{S}_{L}} .
\end{aligned}
$$

The third component of the numerical flux, defined by (18), can be writen under the form

$$
\left[\phi_{i+1 / 2}\right]_{3}=\left[\frac{F_{i}+F_{i+1}}{2}\right]_{3}+\left[\nu_{1}(W)\left(W_{i+1}-W_{i}\right)+\nu_{2}(W)\left(F_{i+1}-F_{i}\right)\right]_{1}+\nu_{3}(W)\left(\psi_{i+1}-\psi_{i}\right),
$$

where

$$
\begin{aligned}
& \nu_{1}(W)=-\frac{1}{2} \frac{\bar{S}_{R} \bar{S}_{L}}{\bar{S}_{R}-\bar{S}_{L}}\left(M_{L} \psi_{i}-M_{R} \psi_{i+1}\right) \\
& \nu_{2}(W)=-\frac{1}{2} \frac{M_{R} \bar{S}_{R} \psi_{i+1}-M_{L} \bar{S}_{L} \psi_{i}}{\bar{S}_{R}-\bar{S}_{L}},
\end{aligned}
$$

and

$$
\nu_{3}(W)=M_{*}\left[\left(\bar{S}_{R} F_{i}-\bar{S}_{L} F_{i+1}+\bar{S}_{L} \bar{S}_{R}\left(W_{i+1}-W_{i}\right)\right)\right]_{(1)} .
$$

Remark 3.1 Observe that the definition of $S_{*}$, the intermediate wave speed, defined by (10), can also be rewritten in function of $\nu_{3}(W)$. We have

$$
S_{*}=\frac{\nu_{3}(W)}{\alpha(W)}, \quad \text { with } \quad \alpha(W)=-M_{*}\left(q_{i+1}-q_{i}-h_{i+1} \bar{S}_{R}+h_{i} \bar{S}_{L}\right) .
$$

Finally, we propose the following extension of the WAF method.

\section{Extension of the WAF method}

For the first and second components, $j=1,2$ :

$$
\begin{aligned}
\phi_{G_{(j)}}^{W A F}=\left[\frac{F_{i}+F_{i+1}}{2}\right. & -\frac{1}{2} \frac{1}{\bar{S}_{R}-\bar{S}_{L}}\left(\left(M_{R} S_{R}-M_{L} S_{L}\right)\left(F_{i+1}-F_{i}-\Delta x G_{i+1 / 2}\right)-\right. \\
& \left.\left.-\bar{S}_{R} \bar{S}_{L}\left(M_{R}-M_{L}\right)\left(W_{i+1}-W_{i}-\Delta x\left(A^{-1} G\right)_{i+1 / 2}\right)\right)\right]_{(j)} .
\end{aligned}
$$


and for the third component:

$$
\begin{aligned}
\phi_{G_{(3)}}^{W A F} & =\left[\frac{F_{i}+F_{i+1}}{2}\right]_{(3)}-\frac{1}{2} \frac{1}{\bar{S}_{R}-\bar{S}_{L}}\left[\left(\bar{S}_{R} M_{R} \psi_{i+1}-\bar{S}_{L} M_{L} \psi_{i}\right)\left(F_{i+1}-F_{i}-\Delta x G_{i+1 / 2}\right)+\right. \\
& +\bar{S}_{L} \bar{S}_{R}\left(M_{L} \psi_{i}-M_{R} \psi_{i+1}\right)\left(W_{i+1}-W_{i}-\Delta x\left(A^{-1} G\right)_{i+1 / 2}\right)+ \\
& \left.+M_{* G}\left(\psi_{i+1}-\psi_{i}\right)\left(\bar{S}_{R} F_{i}-\bar{S}_{L} F_{i+1}+\bar{S}_{L} \bar{S}_{R}\left(W_{i+1}-W_{i}-\Delta x\left(A^{-1} G\right)_{i+1 / 2}\right)\right)\right]_{(1)} .
\end{aligned}
$$

\subsection{Well-balanced property}

In this section we study the well-balanced properties for the purposed extension of the WAF method. This property is related to the order of accuracy of the method.

Due to the approximation of the inverse matrix $A^{-1}$, given by (28), we cannot hope that the scheme balances the stationary solutions up to second order in all the domain $[0, L]$, because we can find a regularity problem when an eigenvalue of matrix $A$ vanishes. So we consider the 'asymptotically well-balanced' property introduced in [10], defined as follows:

Definition 3.1 We say that the scheme (20) is asymptotically well-balanced if there is an increasing sequence of compact sets $\left\{K_{n}\right\}_{n}$ such that:

1. $\mu\left([0, L]-\cup_{n} K_{n}\right)=0$, being $\mu$ the Lebesgue measure in $\mathbb{R}$.

2. For all $n$ there exists a $\delta_{n}>0$ such that if $0<\Delta x<\delta_{n}$, then the scheme balances system (19) up to second order in $K_{n}$.

So we have the following result for the proposed extension of the WAF method:

Theorem 3.1 We consider the scheme (20) with the definitions (21), (22) and (28), then:

i) The scheme is asymptotically well-balanced for all stationary solutions of the SWE.

ii) The scheme preserves exactly the stationary solution of water at rest with arbitrary pollutant concentration.

ProOF:

i) In regular areas for a stationary solution it is verified that $\partial_{x} F=G$, so we obtain that $\frac{F_{i+1}+F_{i}}{2}, \frac{W_{i+1}-W_{i}}{\Delta x}$ and $\frac{F_{i+1}-F_{i}}{\Delta x}$ are approximations of second order for $F_{i+1 / 2}$, $\left(A^{-1} G\right)_{i+1 / 2}$ and $G_{i+1 / 2}$ respectively. For the sake of brevity, we omit the details of the proof that mainly use Taylor expansion (see [12]). So, we focus on the last term of (34),

$$
\left[M_{* G}\left(\psi_{i+1}-\psi_{i}\right)\left(\bar{S}_{R} F_{i}-\bar{S}_{L} F_{i+1}+\bar{S}_{L} \bar{S}_{R}\left(W_{i+1}-W_{i}-\Delta x\left(A^{-1} G\right)_{i+1 / 2}\right)\right)\right]_{(1)} .
$$


If we consider $W$ a stationary solution of SWE with pollutant (19), then it must verify

$$
\partial_{x} q=0, \quad \partial_{x}(q \psi)=0
$$

From where we deduce that $q$ is constant.

In the case of being $q \neq 0$, then $\psi$ is also constant. So the last term of (34) vanishes (because it is multiplied by $\psi_{i+1}-\psi_{i}$ ).

ii) In water at rest solutions, that is, $q=0, \psi$ might not be constant. In this case we have by Taylor expansion following previous arguments that the last term of (34) is at least of second order. In fact, for water at rest we can see that we have exactly

$$
W_{i+1}-W_{i}-\Delta x\left(A^{-1} G\right)_{i+1 / 2}=0 .
$$

So, the last term of (34) vanishes. Moreover we can see that we have exactly

$$
F_{i+1}-F_{i}=G_{i+1 / 2}
$$

With (35) and (36) it is enough to deduce that the scheme exactly balances the stationary solution of water at rest with arbitrary pollutant concentration.

\subsection{Intermediate wave speed}

Note that we obtain the asymptotically well-balanced property independently of the approximation for the intermediate speed, $S_{*}$.

Nevertheless, in the proof of the exact well-balanced property (Theorem $3.1 \mathrm{ii}$ )) for water at rest with arbitrary pollutant concentration, we observe that it is neccesary that the quantity multiplying to $\left(\psi_{i+1}-\psi_{i}\right)$ was zero.

In equation (30), we have denoted the term multiplying to $\left(\psi_{i+1}-\psi_{i}\right)$ by $\nu_{3}$. If we compare (18) and (34), we observe that $\nu_{3}$ must be modified for non-homogeneous systems. Concretely, $\nu_{3}=\nu_{3}(W, G)$, its definitions depends on $G$, the source term.

Moreover in Remark 3.1 we observe that for homogeneous systems $\nu_{3}$ can be written in terms of $S_{*}$, the intermediate speed: $S_{*}=\nu_{3} / \alpha(W)$, with $\alpha(W)$ defined in equation (32).

Finally, in order to be consistent with the fact that the homogeneous WAF method verifies this property, we must modify the approximation of the intermediate wave speed. We denote this new definition by $S_{* G}$, then $S_{* G}=\nu_{3}(W, G) / \alpha(W)$. We obtain:

$$
S_{* G}=\frac{S_{L} q_{i+1}-S_{R} q_{i}-S_{L} S_{R}\left(h_{i+1}-h_{i}-\Delta x\left[\tilde{A}_{i+1 / 2}^{-1} G_{i+1 / 2}\right]_{(1)}\right)}{q_{i+1}-q_{i}-h_{i+1} S_{R}+h_{i} S_{L}} .
$$

\section{$4 \quad$ Numerical tests}

In this section we present several numerical tests. We compare the solution obtained by the WAF method with those obtained by the HLLC solver of first order accuracy and with exact or reference solutions for some tests. It is well known that the HLLC method 
is more diffusive than the WAF method for homogeneous problems. Here we confirm this fact is also true for non-homogeneous cases.

In the first test we study the influence of the approximation of the intermediate wave speed $S_{*}$ or $S_{* G}$ in the WAF method. The second one is a well-balance test, where we check that the scheme preserves water at rest up to the machine accuracy and up to second order a subcritical stationary solution. The third test is an accuracy test proposed in [40] where we add a non-constant pollutant concentration. In the fourth one we perform an 'oscillating lake' test, with a moving shoreline which involves the treatment of dry/wet moving areas. In the fifth numerical test we see a double rarefaction wave producing a dry area. Test 6 consists of a small perturbation of a steady-state solution where the height of the water column is zero in some isolated points. Finally, in test seven we make a comparison with an analytical solution with a shock. We introduce a periodic timevarying boundary condition on the left over the concentration pollutant and we compare the proposed the WAF method, the HLLC solver and the approximated solution built by approximating the characteristic equation for the analytical velocity.

\section{Test 1. Comparing the effect of $S_{*}$ and $S_{* G}$}

This test is performed to show that the same WAF scheme with two different approximations of the intermediate wave speed, $S_{*}$ given by (32) or $S_{* G}$ given by (37) can produce very different results although we obtain a well-balanced scheme for both definitions.

In this test, we effectively observe that considering $S_{* G}$ as the approximation of the intermediate wave speed for the non-homogeneous case is essential for the good behavior of the pollutant concentration.

We set a domain of 4 meters that we have discretized by a constant space step $\Delta x=$ 0.08. We state the CFL condition as 0.9. The final time is $T=1$, and the initial conditions:

$$
h_{0}=18-z_{b} ; \quad q_{0}=h_{0} ; \quad r_{0}=\left\{\begin{array}{ll}
h_{0} & x<2 \\
0 & x \geq 2
\end{array} ; \text { with } z_{b}(x)=\left\{\begin{array}{ll}
4.5 & x<2 \\
0 & x \geq 2
\end{array} .\right.\right.
$$

We compute this test and compare the results when we use the standard speed $S_{*}$ with our new speed $S_{* G}$ for the the WAF method.

We observe that when we use $S_{*}$, we obtain a peak of the pollutant concentration just in $x=2$, the point where there is a gradient in the topography. This is not an unstability, this is the effect of a wrong approximation of the intermediate wave speed. In this problem $S_{*}$ must be always positive, nevertheless the definition of $S_{*}$ produces a negative value just in $x=2$, as it is shown in Figure 2 (a). If we follow this information $\left(S_{*}>0 \forall x \neq 2\right.$, $S_{*}<0$ at $x=2$ ), the scheme must transport the pollutant to the right in all points except for $x=2$ where it must retain the pollutant and transport it to the left. We can see that the scheme does it exactly. In fact, we observe that all the pollutant has been transported until this point and it cannot transverse, what explains the peak observed at Figure 2 (b).

In Figure 2 (a) we compare $S_{*}, S_{* G}$ and the velocity $u=q / h$ at $T=1$. The intermediate wave speed must give an approximation of the velocity in the intercells of the mesh. We observe that $S_{* G}$ is an approximation of the velocity at the intercells, by while $S_{*}$ produces 

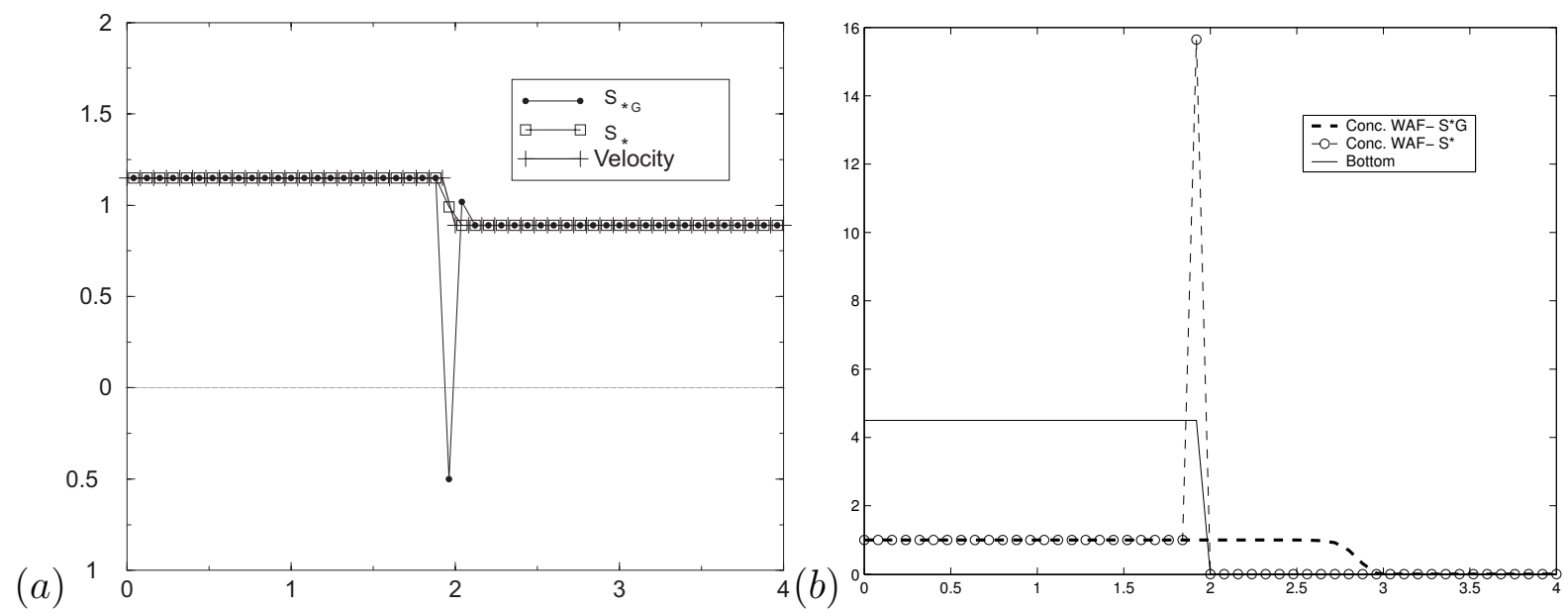

Figure 2: (Test 1). (a) Velocity approximations, $S_{*}$ and $S_{* G}$. (b) Pollutant concentration for the WAF method taking $S_{*}$ or $S_{* G}$.

a great error near the bump. So, from now on we will only consider the approximation of the intermediate wave speed given by $S_{* G}$.

In Figure 3 we present the bottom function, water surface and pollutant concentration provided by the WAF solver and HLLC (see [11]). We observe a less numerical diffusion of the WAF method.

In Table 1, we make a comparison of the runtime (seconds, in an old Pentium II machine) of two methods, WAF and HLLC, in relation to the error for the pollutant concentration in $L^{1}$ norm. For this aim we have solved the problem with meshes $\Delta x=$ $0.08,0.04,0.02,0.01$ and we compare it with a reference solution corresponding to the WAF method with 1000 points. We observe that the runtime is very similar for both methods. However to get the same order of accuracy, for instance an error of 0.02 , the the WAF method spends 1.3 seconds whereas HLLC spends 74.29 seconds. So, in this sense, the WAF method is again better than HLLC.

\begin{tabular}{|c|c|c|c|c|}
\hline$\Delta x$ & $L^{1}$ err WAF & Runtime WAF & $L^{1}$ err HLLC & Runtime HLLC \\
\hline \hline 0.08 & 0.0225 & 1.3 & 0.0834 & 1.21 \\
\hline 0.04 & 0.011 & 4.98 & 0.0584 & 4.87 \\
\hline 0.02 & 0.0047 & 18.51 & 0.0406 & 18.72 \\
\hline 0.01 & 0.0014 & 74.5 & 0.0256 & 74.29 \\
\hline
\end{tabular}

Table 1: (Test 1). Comparison of runtime and error.

\section{Test 2: Well-balanced tests}

We consider two different well-balanced tests. In the first one (Test 2.a) we study the 

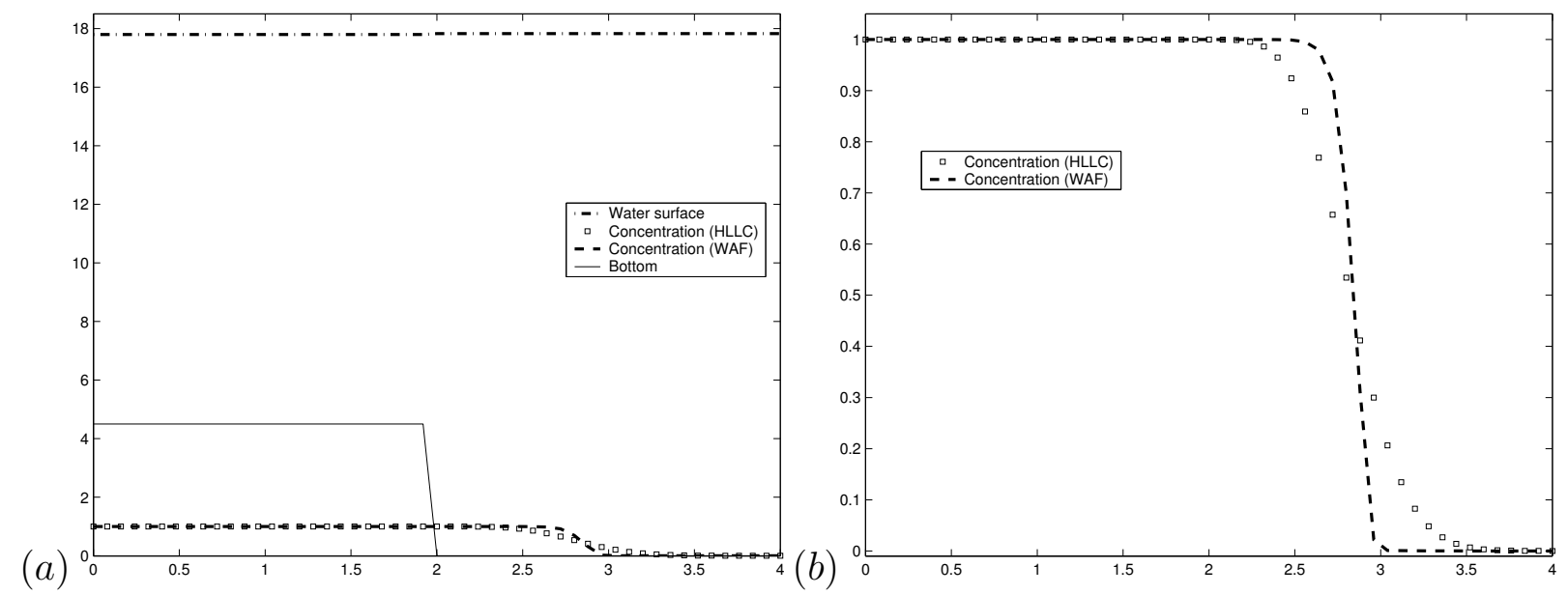

Figure 3: (Test 1). (a) Bottom, water surface and pollutant concentration for HLLC and the WAF method using $S_{* G}$. (b) Pollutant concentrations.

exact well-balanced property of the method for water at rest. In the second one (Test 2.b) we study the second order well-balanced accuracy of the method for a subcritical stationary solution.

\section{Test 2.a: Exact well-balanced property}

We consider a test of water at rest using a random bottom (See Figure 4 (a)). The case of water at rest is the only one of a stationary solution for which the pollutant concentration is also stationary for arbitrary profiles (otherwise, if $q \neq 0, \psi$ must be constant to obtain a stationary solution). Then, we also consider a random profile of $\psi$ (See Figure 4 (b)). We set a domain of $L=1 \mathrm{~m}$. discretized with 100 points and a CFL condition equal to 0.8 . In Table 2 we present the error in $\mathrm{L}^{1}$ norm. The solution is preserved up to the machine accuracy.

\begin{tabular}{|c|c|c|c|c|}
\hline & $h$ & $q$ & $r$, & $\varphi=r / h$ \\
\hline \hline$L^{1}$ error & $3.3827 \times 10^{-16}$ & $1.1725 \times 10^{-15}$ & $1.7493 \times 10^{-15}$ & $1.1328 \times 10^{-15}$ \\
\hline
\end{tabular}

Table 2: (Test 2.a). Errors. Exact well-balanced property.

\section{Test 2.b: Approximated well-balanced property}

In this test we verify numerically the approximated well-balanced property of the WAF method (Theorem $3.1 i$ )).

We consider a subcritical stationary solution. The length of the domain is $20 \mathrm{~m}$. The discharge is constant, $q=4.42$, and the bottom function

$$
z_{b}(x)=0.2 \mathrm{e}^{-0.16(x-10)^{2}}, \quad x \in[0,20] .
$$



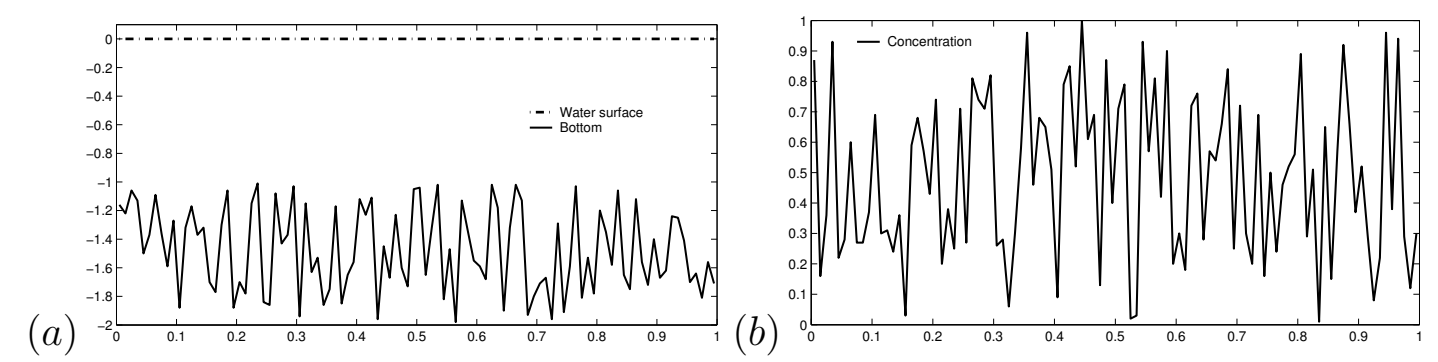

Figure 4: (Test 2.a). (a) Random bottom, (b) Random concentration of pollutant

The analytical solution for $h(x)$ can be obtained as follows. We have that the equation for the velocity $u=q / h$ is

$$
\partial_{t}(u)+\partial_{x}\left(\frac{u^{2}}{2}+g\left(h+z_{b}\right)\right)=0 .
$$

Then, for a regular stationary solution, $u^{2} / 2+g\left(h+z_{b}\right)=k$, with $k$ a constant value. If we rewrite this equation in terms of $h$ and $q$, we obtain that $h$ is a root of

$$
P(h)=h^{3}+\left(z_{b}-\frac{k}{g}\right) h^{2}+\frac{q^{2}}{2 g} .
$$

To define $k$, we impose at $x=0$ that $h=2-z_{b}(0)$. The subcritical solution correspond with the bigger root of $P(h)$.

In Table 3 we observe that effectively we achived the second order accuracy.

\begin{tabular}{|c|c|c|c|c|}
\hline Nodes & $L^{1}$ err $h$ & $L^{1}$ order $h$ & $L^{1}$ err $q$ & $L^{1}$ order $q$ \\
\hline \hline 20 & $1.58 \times 10^{-3}$ & - & $5.02 \times 10^{-3}$ & - \\
\hline 40 & $5.07 \times 10^{-4}$ & 1.646 & $1.21 \times 10^{-3}$ & 2.0513 \\
\hline 80 & $1.3 \times 10^{-4}$ & 1.967 & $3.04 \times 10^{-4}$ & 1.9965 \\
\hline 160 & $3.2 \times 10^{-5}$ & 1.995 & $7.6 \times 10^{-5}$ & 1.9985 \\
\hline 320 & $9 \times 10^{-6}$ & 1.904 & $1.9 \times 10^{-5}$ & 1.9987 \\
\hline
\end{tabular}

Table 3: (Test 2.b). Errors and order. Subcritical stationary solution.

\section{Test 3: Order of accuracy}

We consider a test proposed in [40] to study the order of accuracy of the method. Although we also introduce a non constant profile of the concentration of pollutant. We compute up to $t=0.1$ where any shock still appears in any of the unknowns. The domain is $[0,1]$, we impose periodic boundary conditions and initial conditions

$$
\begin{gathered}
h(x, 0)=5+\mathrm{e}^{\cos (2 \pi x)}, \quad q(x, 0)=\sin (\cos (2 \pi x)), \\
\psi(x, 0)=\frac{1}{2}(\cos (4 \pi x)+1) .
\end{gathered}
$$


The bottom functions is defined by

$$
z_{b}(x)=\sin ^{2}(\pi x)
$$

As in this case we do not know an analytical solution for the problem, we compute a reference solution with the WAF method using 12800 cells to compute the errors. In all cases we fix the CFL condition to 0.8 .

In Table 4 we present the results for the errors in norm $L^{1}$ and the corresponding order. The second order of accuracy is achieved.

\begin{tabular}{|c|c|c|c|c|c|c|}
\hline Nodes & $L^{1}$ err $h$ & $L^{1}$ order $h$ & $L^{1}$ err $q$ & $L^{1}$ order $q$ & $L^{1}$ err $r$ & $L^{1}$ order $r$ \\
\hline \hline 25 & $2.802 \times 10^{-2}$ & - & $3.143 \times 10^{-1}$ & - & $2.112 \times 10^{-2}$ & - \\
\hline 50 & $1.021 \times 10^{-2}$ & 1.456 & $9.702 \times 10^{-2}$ & 1.695 & $7.464 \times 10^{-3}$ & 1.501 \\
\hline 100 & $3.228 \times 10^{-3}$ & 1.661 & $2.677 \times 10^{-2}$ & 1.857 & $2.533 \times 10^{-3}$ & 1.559 \\
\hline 200 & $9.15 \times 10^{-4}$ & 1.819 & $6.594 \times 10^{-3}$ & 2.021 & $7.51 \times 10^{-4}$ & 1.753 \\
\hline 400 & $2.53 \times 10^{-4}$ & 1.851 & $1.553 \times 10^{-3}$ & 2.085 & $2.07 \times 10^{-4}$ & 1.858 \\
\hline
\end{tabular}

Table 4: (Test 3). Errors and order.

\section{Test 4: Oscillating lake}

In this test we study an oscillating lake over a non flat bottom. This test has been proposed by Audusse et al in [1]. A shore line variable in time appears in the problem.

The initial conditions are

$$
\begin{gathered}
h(x, 0)=\max \left(0,0.4-z_{b}(x)+0.04 \sin ((x-0.5) / 0.25)+0.04 \max \left(0 .,-0.4+z_{b}(x)\right)\right), \\
q(x, 0)=0, \quad z_{b}(x)=\frac{1}{2}\left(1-\frac{1}{2}\left(\cos \left(\frac{\pi(x-0.5)}{0.5}\right)+1\right)\right) .
\end{gathered}
$$

The length of the domain is $L=1 \mathrm{~m}$., we consider 200 points and a CFL condition equal to 0.8 .

In Figure 5 the water surface is plotted at the initial condition and at $t=19.87 \mathrm{~s}$, corresponding to a period where the wave is located at the opposite side of the initial condition.

\section{Test 5: Small perturbation of a steady-state solution}

This test has been proposed by Kurganov and Petrova in [17]. It consists of studying a small perturbation in the water surface for a steady-state solution, where the height of the water column is zero in some isolated points.

The domain is $[0,1]$, we compare the approximations obtained with 200 points and 3200 points. The CFL condition is set to 0.8 . The initial conditions are

$$
h(x, 0)=\left\{\begin{array}{ll}
1+\varepsilon-z_{b}(x) & 0.1<x<0.2 \\
1-z_{b}(x) & \text { otherwise }
\end{array} \quad q(x, 0)=0,\right.
$$




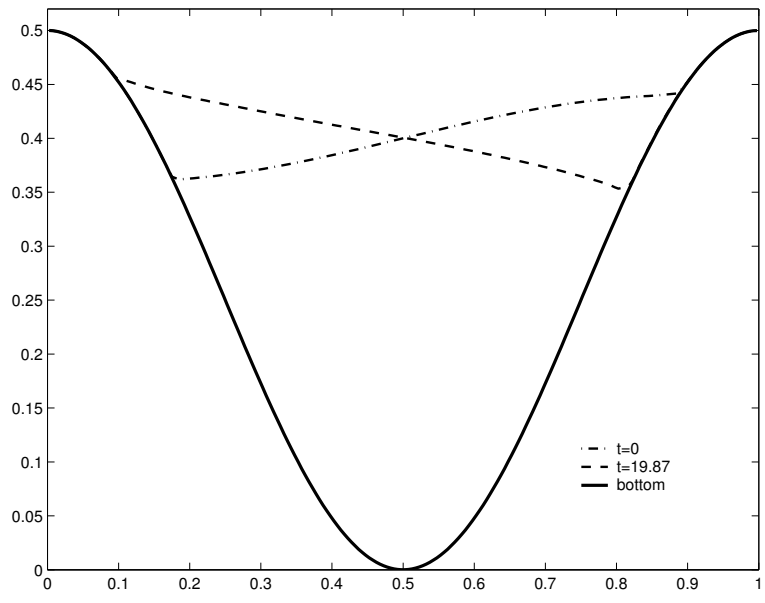

Figure 5: (Test 4). Oscillating lake. Water surface at $t=0$ and $t=19.87$.

where $\varepsilon=0.001$ is the small perturbation on the water surface located between 0.1 and 0.2 . The bottom function is defined by

$$
z_{b}(x)= \begin{cases}10(x-0.3), & 0.3 \leq x \leq 0.4 \\ 1-0.0025 \sin ^{2}(25 \pi(x-0.4)), & 0.4 \leq x \leq 0.6 \\ -10(x-0.7), & 0.6 \leq x \leq 0.7 \\ 0 & \text { otherwise }\end{cases}
$$

In Figure 6 we present the initial conditions. In Figure 7 we present a zoom on $[0,1] \times$ $[0.995,1.005]$ of the solution for $t=1 \mathrm{~s}$. computed with 200 points. In Figure 8 we make a comparison with the solution for 3200 points.

In Figure 8 (a) we present a zoom on the first point of jump of the bottom found by the wave. We have a vertical wall at $x=0.4$, but in discret we have a plane with slope $1 / \Delta x$. That explains the difference in this area between both values of $\Delta x(\Delta x=1 / 200$ and $\Delta x=$ 1/3200). Otherwise, in Figure 8 (b) we present a zoom on $[0.25,0.75] \times[0.9994,1.0005]$ of the water surface. We observe that the results obtained with 200 and 3200 points are not so different in the intermediate areas between the two vertical walls. And as we have previously explained, it is greater close to the vertical walls at $x=0.4$ and $x=0.6$.

\section{Test 6: Dry zone by a double rarefaction wave}

This test was proposed by Gallouët in [14]. It consists of a double rarefaction wave (see [32]) including an obstacle at the bottom. The numerical treatment of dry/wet areas has been done following the technique described in [6].

The length of the domain is 20 meters and the space step $\Delta x=0.1$. We set the CFL condition equal to 0.8 . The initial conditions are

$$
h_{0}=10-z_{b} ; \quad q_{0}=\left\{\begin{array}{ll}
350 & x>50 / 3 \\
-350 & \text { otherwise }
\end{array} ; \quad r_{0}=\left\{\begin{array}{ll}
h_{0} & 25 / 3<x<12.5 \\
0 & \text { otherwise }
\end{array} ;\right.\right.
$$



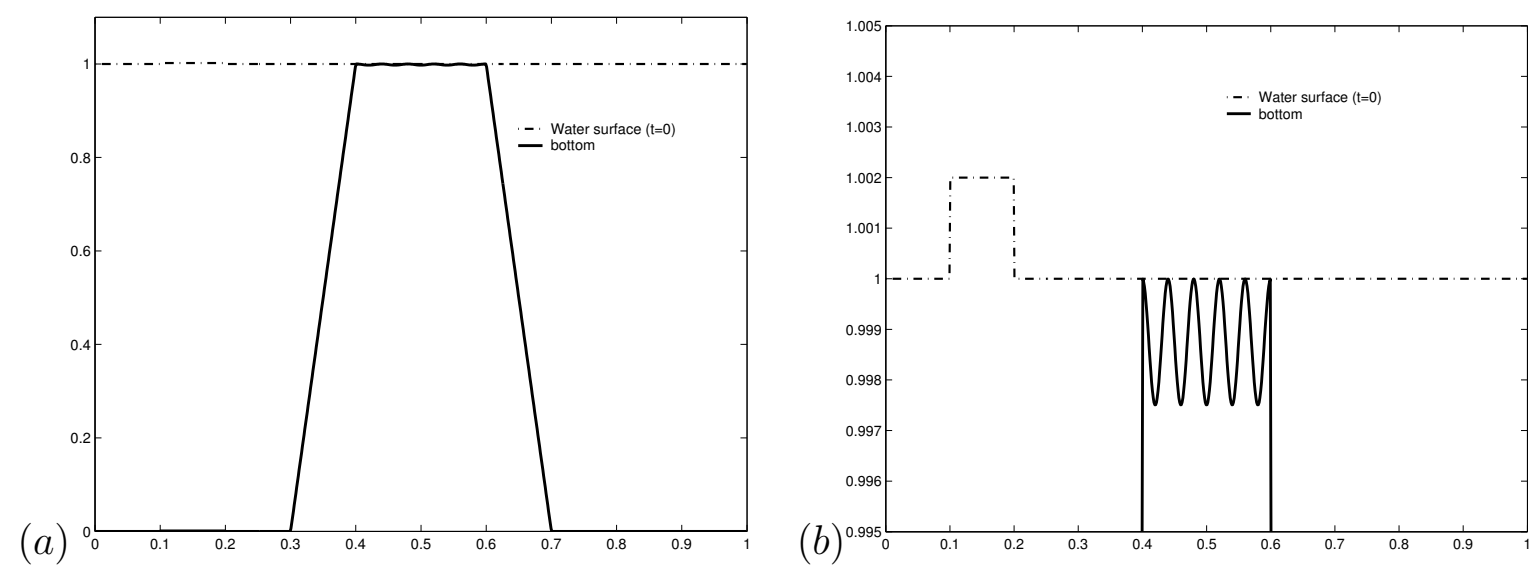

Figure 6: (Test 5). Initial condition. Water surface and bottom. (b) zoom on $[0,1] \times$ $[0.995,1005]$

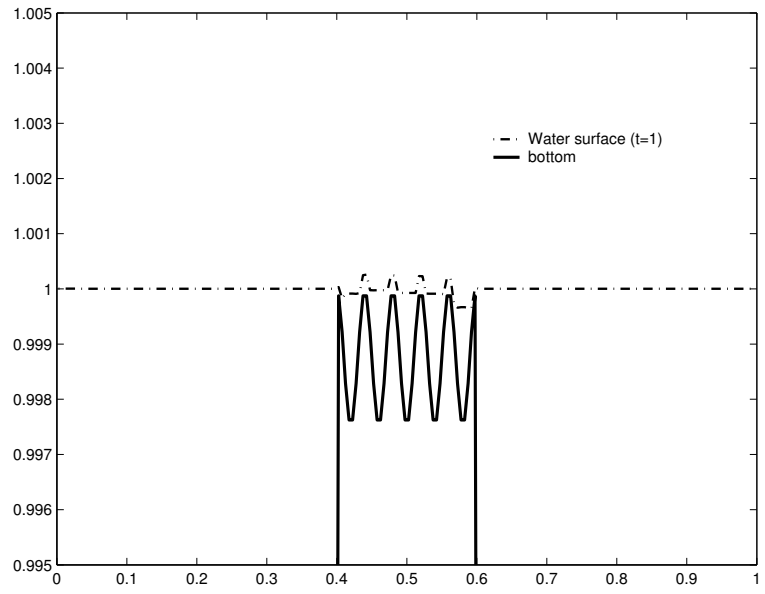

Figure 7: (Test 5). Solution for $t=1 \mathrm{~s}$. Zoom on $[0,1] \times[0.995,1005]$ 

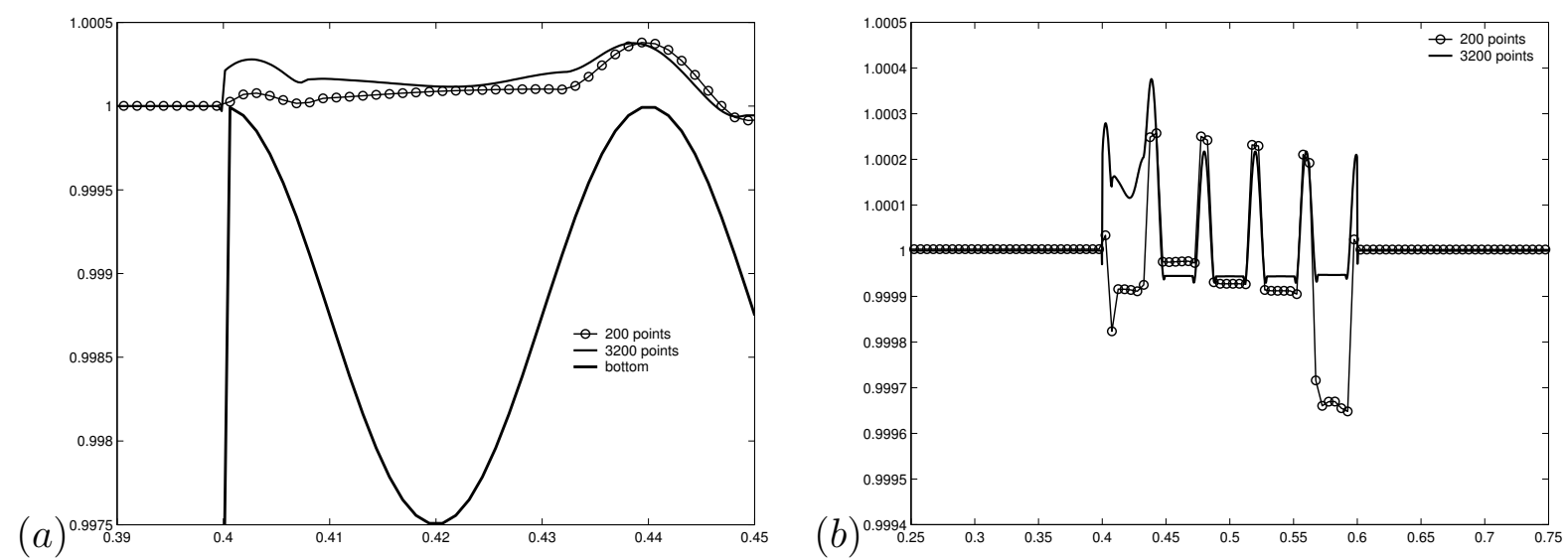

Figure 8: (Test 5). Line with circles: 200 points. Continuous line: 3200 points. (a) Zoom on $[0.39,0.45] \times[0.9975,1.0005]$, Water surface and bottom. (b) Zoom on $[0,25,0,75] \times$ $[0.9994,1.0005]$, Water surface

$$
\text { with } z_{b}(x)=\left\{\begin{array}{ll}
1 & 25 / 3<x<12.5 \\
0 & \text { otherwise }
\end{array}\right. \text {. }
$$

In Figure 9 we compare the water surface obtained by WAF and HLLC with a reference solution computed with the WAF method with a mesh of 3000 points. We observe how the WAF method better captures the shock at $x=25 / 3$, and the bump on the water surface around $x=6.5$. In Figure 9 (b) we show the solution for the pollutant concentration at time $T=0.18$, again we see that the WAF method introduces a less numerical diffusion.

Test 7: Stationary transcritical flux with a shock and a periodic time-varying pollutant concentration

We consider a classical test for a stationary solution for $h$ and $q$ but including in this case a periodic time pollutant source as a boundary condition.

The domain length is 20 meters and the bottom function is defined by a bump at the middle of the domain:

$$
z_{b}(x)=\left\{\begin{array}{ll}
0.2-0.05(x-10)^{2} & 8<x<12 \\
0 & \text { otherwise }
\end{array} .\right.
$$

We consider a space step $\Delta x=0.1$ and a CFL condition equal to 0.9.

The initial conditions are:

$$
h_{0}=0.33-z_{b} ; \quad q_{0}=0 ; \quad r_{0}=0,
$$

and the boundary conditions $q=0.18$ and $r=r_{p}(t)$ at $\mathrm{x}=0 ; h=0.33$ at $x=20$. The function $r_{p}$ is a periodical function in time that represents a contribution of pollutant of three seconds every ten seconds; and it is given by:

$$
r_{p}(t)=\left\{\begin{array}{ll}
h & t \in[10 i, 10 i+3] \\
0 & \text { otherwise }
\end{array}, \quad \text { for } i \in \mathbf{N} .\right.
$$



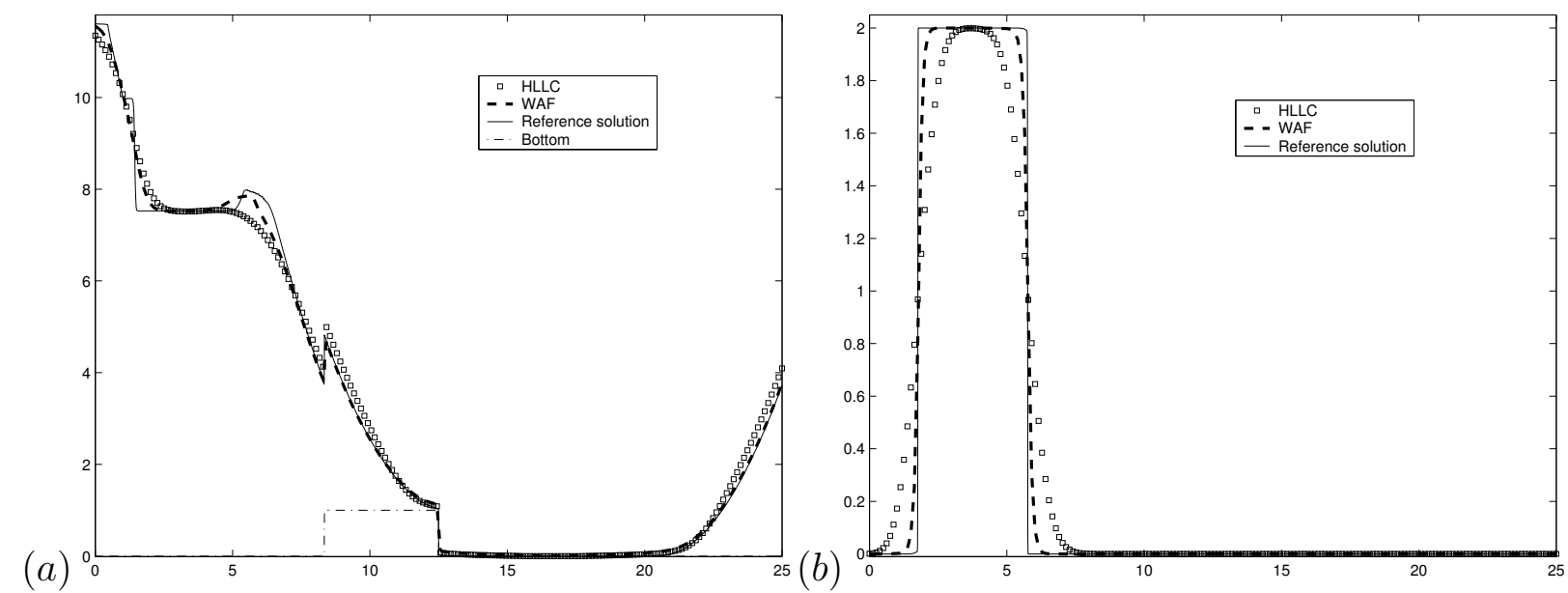

Figure 9: (Test 6). (a) Water surface at $T=0.25$. (b) Pollutant concentration at $T=0.18$.

For this test the analytical solution for $h$ and $q$ can be obtained. $q$ is constant and equals to the boundary condition, so $q=0.18$. To obtain $h$, it is necessary to solve the third order polynomial

$$
P(h)=h^{3}+\left(z_{b}-\frac{k}{g}\right) h^{2}+\frac{q^{2}}{2 g}
$$

where $k$ is the constant energy value and $g$ is the gravity. This solution has a shock that connects two different constant values of the energy. The position of the shock is obtained by the Rankine-Hugoniot condition.

With this data we can calculate the velocity of the fluid $u=q / h$. So we can predict the position of the pollutant fronts by approximating the trajectory of the points, by solving the characteristic curves problem

$$
\left\{\begin{array}{l}
\dot{x}(t)=u(x(t), t) \\
x(0)=0
\end{array}\right.
$$

To approximate $x(t)$ we have used a Runge-Kutta method of third order. Once we have $x(t)$, we can build the profile of the pollutant concentration, that is a passive scalar transported with the velocity of the fluid.

In Figure 10, we compare the exact solution for $h$ and $q$ with the numerical results obtained with the proposed the WAF method. In Figure 10 (a) we compare the water surface. This is a transcritical problem with a critical point at $x=10$. We observe that the method does not produce the phenomenon known as 'dog leg' as presented in Roe method. In Figure 10 (b) the velocity of the fluid is compared. Usually the discharge value is presented for this test but we are interested in the velocity, which is the responsible for the transport of the pollutant concentration. We see that the approximated velocity suits the analytical solution, even close to the shock. 

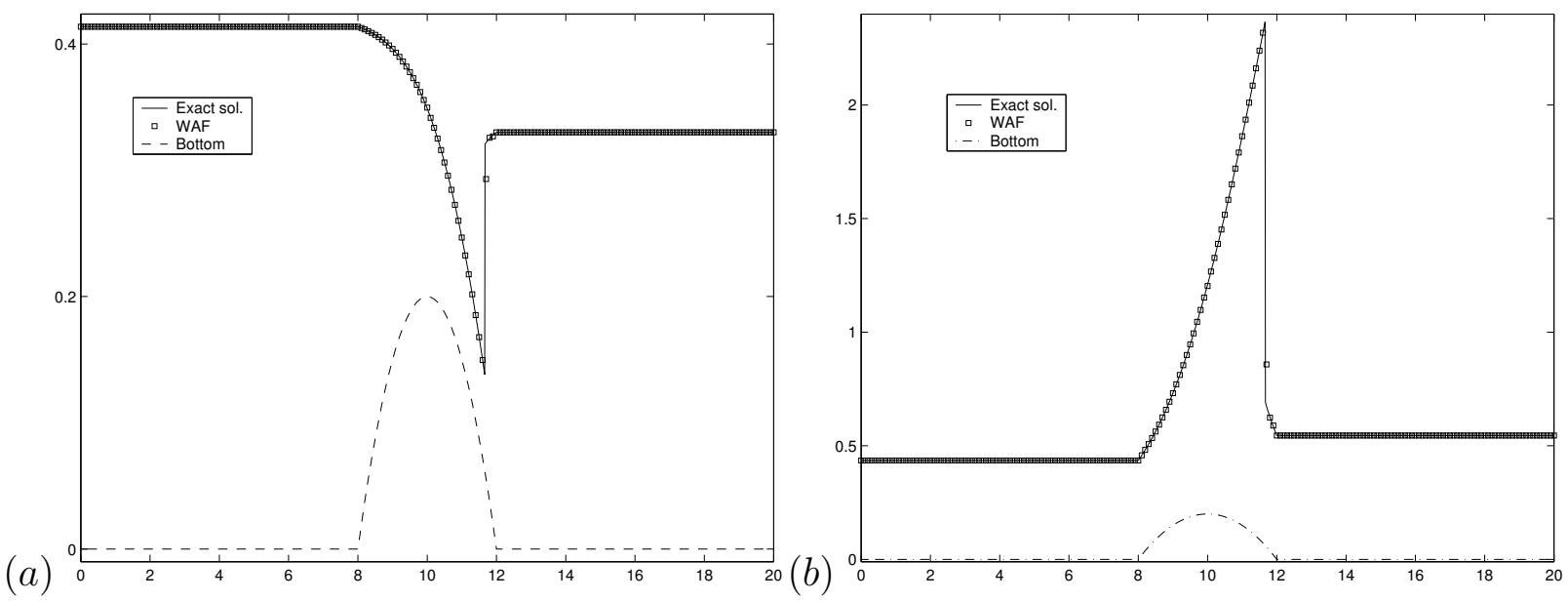

Figure 10: (Test 7). (a) Water surface and topography. (b) Velocity.

To compare the pollutant concentration obtained by the WAF and HLLC solver with the exact solution, we begin at $t=189$, a time that ensures the solution for $h$ and $q$ to be stationary.

In Figure 11 we compare the pollutant concentration for the WAF and HLLC solver for different times values. We observe the great numerical diffusion introduced by the HLLC solver in comparison with the WAF method. In Figure 12 we compare the WAF method with the approximated solution computing solving (38). The considered times show the transition of a column of pollutant through the bump where the velocity of the fluid grows.

One of the characteristics of the solution for the pollutant concentration is that the width of the rectangles of pollutant that is introduced by the right boundary is not the same in all the domain. That is because the velocity is not constant in all the domain, then the velocity of the particle at the front of the rectangle is different to those at the back.

If we look at Figure 10 (b) where the velocity is drawn, we observe two regions of constant velocity and a fast increase of the velocity over the bump until the shock. Then, according to this profile of velocity we can see how the rectangle of pollutant expands in this zone in Figures 11 and 12.

In Figure 12 we observe that the biggest difference between the numerical and the exact solution just corresponds to time $t=195$, over the area of bigger velocity previous to the shock (check the velocity profile of Figure 10 (b)). This difference at the back of the rectangle after the bump is motivated by the numerical diffusion of the scheme because some points have not still pass through the shock. We observe that after the shock, in the zone of constant velocity, this difference between exact and numerical solution is reduced. 

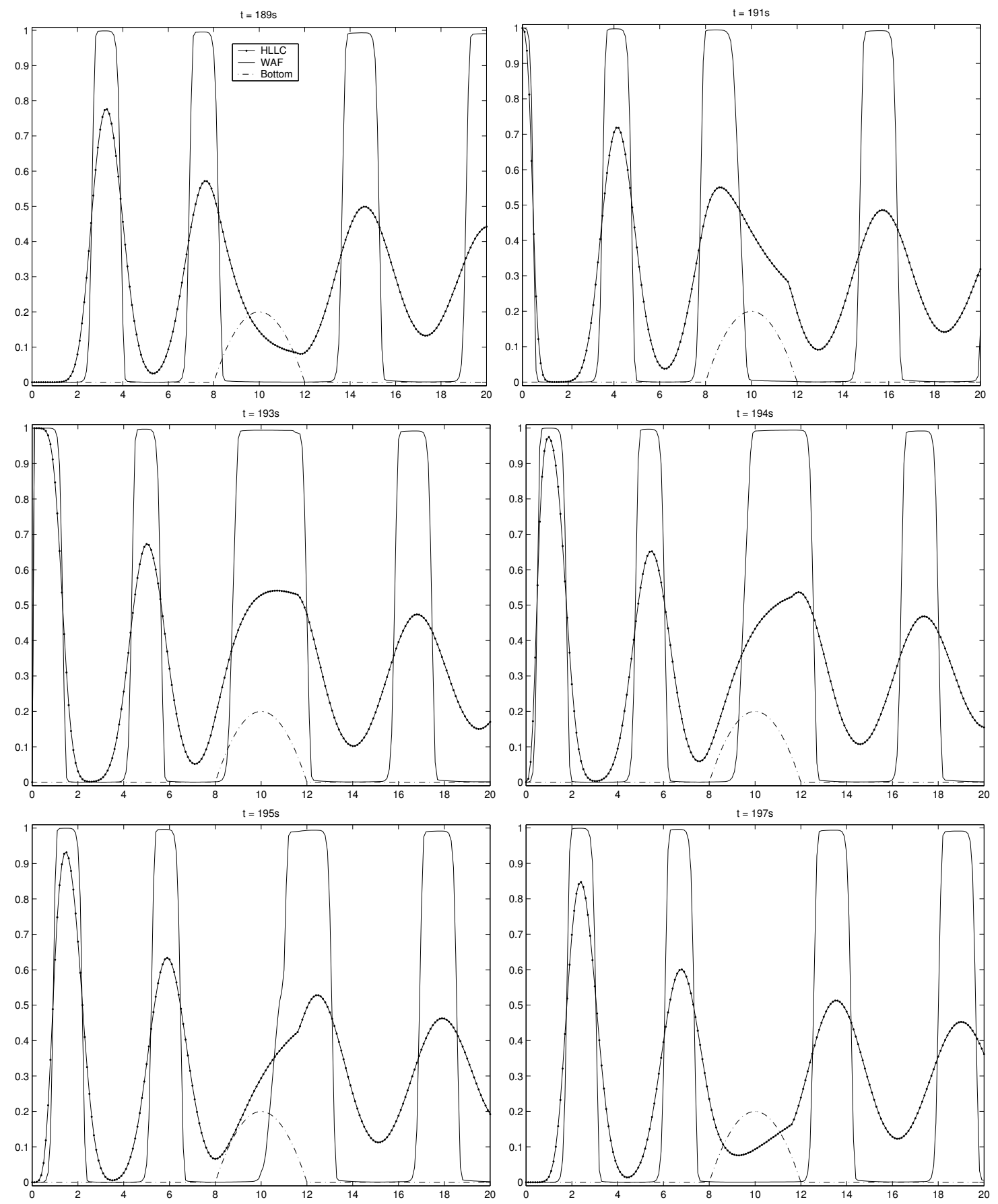

Figure 11: (Test 7). Concentration for WAF and HLLC methods. 

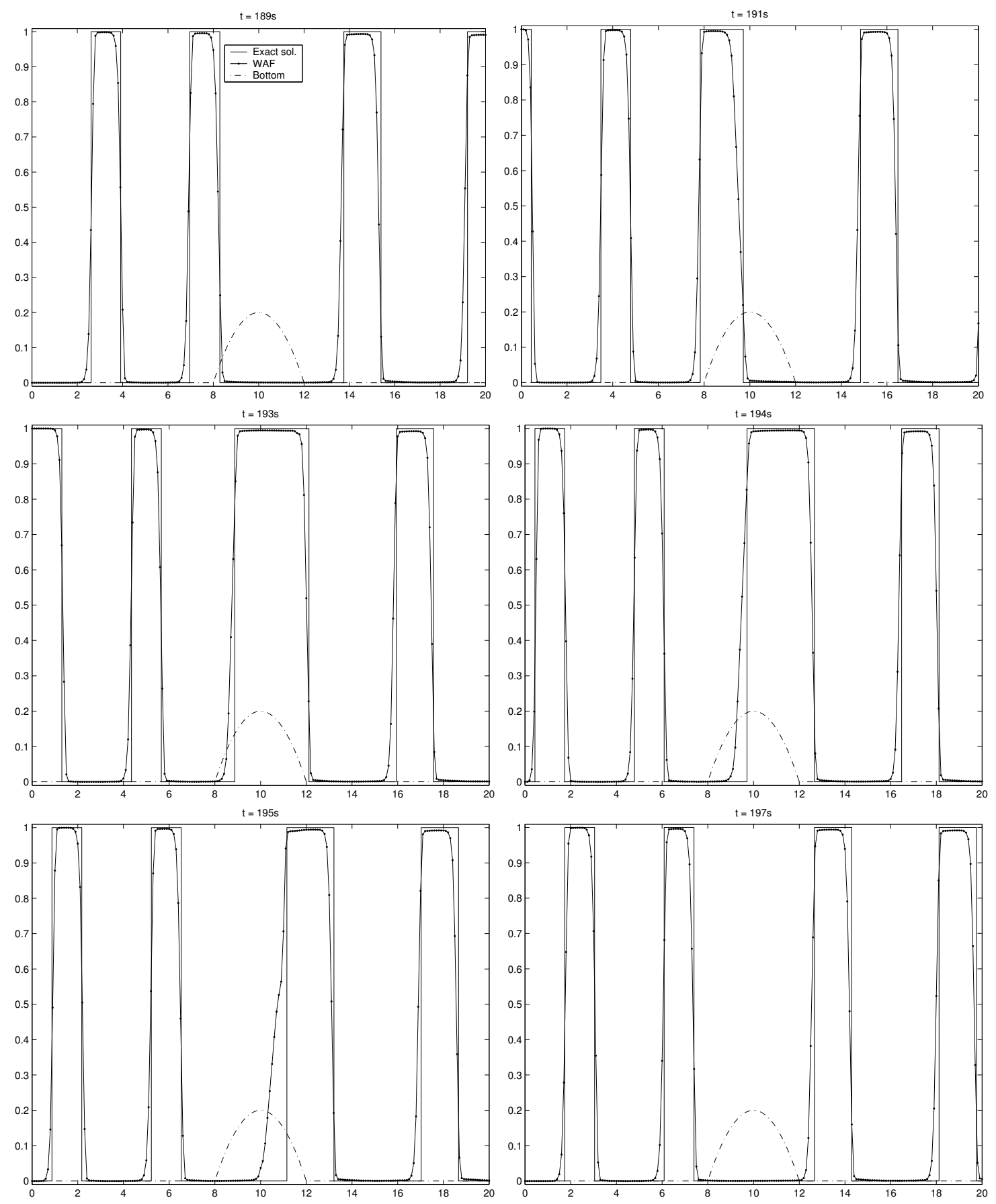

Figure 12: (Test 7). Comparing WAF approximation with exact solution. 


\section{Conclusions}

In this paper we have proposed an extention of two versions of the WAF method to non-homogeneous Shallow Water equations with pollutant. For the homogeneous case we rewrite the third component of the flux that approximates the mass concentration, in terms of an approximation of the intermediate wave speed. In order to obtain the same relations for the non-homogeneous case we observe that the approximation of the intermediate wave speed must also be modified depending on the source term. The scheme is asymptotically well-balanced (see [10]) and exactly preserves water at rest with arbitray concentration of pollutant. Finally we have performed several tests where we study numerically the well-balanced properties of the model, we present a test of order, three tests with dry/wet transitions and comparison with an analytical solution.

\section{Acknowledgements}

This work has been partially supported by the Spanish Government Research project MTM2006-01275.

\section{References}

[1] E. Audusse, F. Bouchut, M.-O. Bristeau, R. Klein, B- Perthame, A fast and stable well-balanced scheme with hydrostatic reconstruction for shallow water flows, SIAM J. Sci. Comput,. 25(6): 2050-2065 (electronic), 2004.

[2] A. Bermúdez - M. E. Vázquez Cendón, Upwind Methods for Hyperbolic Conservation Laws with Source Terms. Computers Fluids 23-8 1049-1071 (1994).

[3] S.J. Billet, E.F. Toro, On WAF-Type Schemes for Multidimensional Hyperbolic Conservation Laws, J. Comput. Phys. 130, 1-24 (1997).

[4] F. Bouchut, Nonlinear Stability of Finite Volume Methods for Hyperbolic Conservation Laws and Well-Balanced Schemes for Sources. Birkäuser Verlag (2004).

[5] C. Berthon, F. Marche, A positive preserving high order VFROE scheme for Shallow Water Equations. A Class of relaxation schemes. Submitted (2007)

[6] M.J. Castro, A.M. Ferreiro, J.A. García, J.M. González, J. Macías, C. Parés, M.E. Vázquez. On the numerical treatment of wet/dry fronts in shallow flows: applications to one-layer and two-layer systems. Math. Comp. Model. 42 (3-4): 419-439 (2005).

[7] M.J. Castro, J.M. González-Vida, C. Parés, Numerical treatment of wet/dry fronts in shallow flows with a modified Roe scheme. Math. Mod. Meth. App. Sci. Vol. 16, No. 6, 897-931 (2006). 
[8] T. Chacón Rebollo, E.D. Fernández-Nieto, M. Gómez Mármol A flux-splitting solver for shallow watter equations with source terms . Internat. J. Numer. Methods Fluids, vol. 42, n1, pp.23-55, 2003.

[9] T. Chacón Rebollo, A. Domínguez Delgado, E.D. Fernández Nieto, An entropycorrection free solver for non-homogeneous shallow water equations. To appear in Mathematical Modelling and Numerical Analysis, M2AN.

[10] T. Chacón Rebollo, A. Domínguez Delgado, E.D. Fernández-Nieto, Asymptotical balanced schemes for non-homogeneous hyperbolic systems. Aplications to Shallow Water equations, C.R. Acad. Sci.Paris. 338, 85-90. (2004).

[11] E.D. Fernández-Nieto, D. Bresch, J. Monnier, A consistent intermediate wave speed for a well-balanced HLLC solver. Submitted to C.R. Acad. Sci.Paris (2007)

[12] E.D. Fernández Nieto: Aproximación numérica de leyes de conservación hiperbólicas no homogéneas. Aplicación a las ecuaciones de aguas someras. Ph. D. Thesis Universidad de Sevilla, (2003).

[13] J.M. Gallardo, C. Parés, M.J. Castro, On a well-balanced high-order finite volume scheme for shallow water equations with topography and dry areas. J. Comput. Phys. 227, 574-601 (2007).

[14] T. Gallouët; J.M. Hérard; N. Seguin: Some approximate Godunov schemes to compute shallow water equations with topography, Comput. Fluids, vol. 32, n4, pp. 479-513.

[15] L.Gosse. A well-balanced scheme using non-conservative products designed for hyperbolic system of conservation laws with source terms. Mat. Mod. Meth. Appl. Sc. 11, 339-365 (2001).

[16] J.M. Greenberg, A. Y. Leroux. A Well-Balanced scheme for the numerical processing of source terms in hyperbolic equations.. SIAM J. Numer. Anal. Vol. 33 No. 1, pp. 1-16 (1996).

[17] A. Kurganov, G. Petrova. A second-order well-balanced positivity preserving centralupwind scheme for the Saint-Venant system, Commun. Math. Sci., 5(1): 133-160, 2007.

[18] P.G. LeFloch. Shock waves for nonlinear hyperbolic systems in nonconservative form. Institute for Math. and its Appl., Minneapolis, Preprint 593, (1989).

[19] Randall J. LeVeque Balancing Source Terms and Flux Gradients in High-Resolution Godunov Methods: The Quasi-Steady Wave-Propagation Algorithm. J. Comput. Phys. 146, 346-365 (1998). 
[20] F. Marche, P. Bonneton, P. Fabrie, N. Seguin. Evaluation of well-balanced borecapturing schemes for $2 D$ wetting and drying processes, Internat. J. Numer. Methods Fluids, 53(5): 867-894, 2007.

[21] C.Parés, M.J.Castro. On the well-balanced property of Roe's method for nonconservative hyperbolic systems. Applications to shallow-water systems. ESAIM: M2AN, 38(5) 821-852 (2004).

[22] B. Perthame, C. Simeoni, A kinetic scheme for the Saint-Venant system with a source term. Calcolo 38 n. 4, 201-231 (2001).

[23] P.L. Roe, Upwind differencing schemes for hyperbolic conservation laws with source terms. Nonlinear Hyperbolic Problems, C. Carraso, P.-A. Raviart and D. Serre, eds., Springer-Verlag, Lecture Notes in Matematics 1270, 41-51 (1986).

[24] T. Schwartzkopff, C.D. Munz, E.F. Toro, ADER: High-Order Approach for Linear Hyperbolic Systems in 2D, Journal of Scientific Computing, Vol. 17, Nos 1-4, pp 231$240,2002$.

[25] V.A. Titarev, E.F. Toro, WENO schemes based on upwind and centered TVD fluxes, Computers and Fluids, Vol. 34, pp 705-720, 2005.

[26] V.A. Titarev, E.F. Toro, Analysis of ADER and ADER-WAF schemes, IMA J. Num. Anal. vol. 27(3), pp. 616-630, 2007.

[27] V.A. Titarev and E.F. Toro, ADER Schemes for three-dimensional non-linear hyperbolic systems. J. Com. Phys, Vol. 204, pp 715-736, 2005.

[28] V.A. Titarev, E.F. Toro ADER: Arbitrary High. Order Godunov Approach, Journal of Scientific Computing, Vol. 17, Nos 1-4, pp 609-618, 2002.

[29] E.F. Toro: A weighted average flux method for hyperbolic conservation laws, Proc. R. Soc. Lond. A 423, 401-418 (1989).

[30] E.F. Toro, Riemann problems and the the WAF method for solving two-dimensional shallow water equations, Phil. Trans. Roy. Soc. London, A338, pp.43-68, 1992.

[31] E.F. Toro, The weighted average flux method applied to the time dependent euler equations, Phil. Trans. Roy. Soc. London, A341, pp.499-530, 1992.

[32] E.F. Toro: Shock-Capturing Methods for Free-Surface Shallow Flows, Wiley, England (2001).

[33] E. F. Toro, R. C. Millington, L.A.M. Nejad, Primitive upwind numerical methods for hyperbolic partial differential equations, in Bruneau, C. H. (ed.), Sixteenth International Conference on Numerical Methods for Fluids Dynamics. Lecture notes in physics, Springer-Verlag, Berlin, pp. 421-426, 1998. 
[34] E.F. Toro, V.A. Titarev, Solution of the Generalised Riemann Problem for AdvectionReaction Equations, Proc. Royal Society of London A Vol. 458, pp 271-281, 2002.

[35] E.F. Toro, V.A. Titarev, TVD Fluxes for the High-Order ADER Schemes, J. Sci. Computing, vol. 24, n3, pp. 285-309, 2005.

[36] E.F. Toro, V.A. Titarev, ADER schemes for scalar hyperbolic conservation laws with source terms in three space dimensions, J. Com. Phys, Vol. 202, pp 196-215, 2005.

[37] E.F. Toro and V.A. Titarev, ADER schemes for scalar hyperbolic conservation laws with source terms in three space dimensions, J. Com. Phys, Vol. 202, pp 196-215, 2005.

[38] I. Toumi, A weak formulation of Roe's approximate Riemann Solver. J. Comp. Phys. 102(2): 360-373, (1992).

[39] G. Vignoli, V.A. Titarev, E.F. Toro, ADER schemes for the shallow water equations in channel with irregular bottom elevation, Submitted to Elsevier Science.

[40] Yulong Xing, Chi-Wang Shu, High order well-balanced finite volume WENO schemes and discontinuous Galerkin methods for a class of hyperbolic systems with source term J. Comput. Phys. 214, 567-598 (2006).

[41] J.G. Zhou, D.M. Causon, C.G. Mingham, and D. M. Ingram, The Surface Gradient Method for the Treatment of Source Terms in the Sallow-Water Equations J. Comput. Phys. 168,1-25 (2001). 